Pacific Journal of Mathematics

EXTENSION OF ACTIONS ON STIEFEL MANIFOLDS 


\title{
EXTENSION OF ACTIONS ON STIEFEL MANIFOLDS
}

\author{
IsABel Dotti de Miatello
}

\begin{abstract}
It is natural to ask for examples of $\pi$-biaxial actions in the unitary and simplectic case which do not come from the orthogonal case. Here we provide examples of such actions.
\end{abstract}

Introduction. Let us consider the left translation action of $U(n)(S p(n))$ on the Stiefel manifold $U(n+k+2) / U(n+k)(S p(n+$ $k+2) / S p(n+k)), k$ nonnegative integer, $n \geqq 2$.

The main result to be proved here is that the above action can not be extended to a biaxial $O(2 n)(U(2 n))$ action. The proof uses strongly the correspondence between $U(n)(S p(n)) \pi$-biaxial manifolds with orbit space diffeomorphic to a disk and framed submanifolds of the sphere.

The main references for this article are the book Introduction to Compact Transformation Groups (Bredon [3]) for the general theory of groups actions and the mimeographed notes (Bredon [4]) Biaxial Actions of the Classical Groups for the classification of such actions and characterization of restrictions of $\pi$-biaxial manifolds.

1. Preparatory material. Let $G$ be a compact Lie group and $\sigma: G \rightarrow G l(V)$ be a representation of $G$ on the real vector space $V$. By a $G$-manifold "modeled on $\sigma$ " we mean a smooth $G$ manifold such that each orbit in $M$ has an open invariant neighborhood which is equivariantly diffeomorphic to an open invariant set in the representation space $V$ of $\sigma$.

Let $d=1,2,4$. In these three cases we let $G_{n}^{d}$ stand for $O(n)$, $U(n)$ or $S p(n)$. The standard representation of $G_{n}^{d}$ on $R^{n d}$ will be denoted by $\sigma_{n}$ and the trivial real $k$-dimensional representation by $\theta_{k}$. A $G$-manifold $M$ is modeled on $2 \sigma_{n}+\theta_{k} k<0$ if $M \times R^{-k}$ is modeled on $2 \sigma_{n}\left(G_{n}^{d}\right.$ acts trivially on $\left.R^{-k}\right)$.

Definition 1.1. A $G_{n}^{d}$ manifold $M, n \geqq 2$, will be called biaxial if it is modeled on the representation $2 \sigma_{n}+\theta_{k}$. It is not hard to see that a $G_{n}^{d}$ manifold is biaxial iff the following four conditions hold:

1. The principal orbit type is $G_{n}^{d} / G_{n-2}^{d}$. The other orbit types (if any) are $G_{n}^{d} / G_{n-1}^{d}$ and fixed points (if any).

2. The representation of $G_{n}^{d}$ about a fixed point is $2 \sigma_{n}+\theta_{k}$.

3. The slice representation of $G_{n-1}^{d}$ on the normal space to an orbit at a point with isotropy group $G_{n-1}^{d}$ is $\sigma_{n-1}+\theta_{k+d+1}$. 
4. The slice representation of $G_{n-2}^{d}$ normal to a principal orbit is $\theta_{k+d+2}$.

Suppose now that $K$ is a closed subgroup of $G, G$ a compact Lie group, $M$ a $G$-manifold and $M_{(K)}$ the set of points on orbits of type $G / K$. Under this hypothesis it is proved in Bredon [3] that the orbit map $M_{(K)} \rightarrow M_{(K)} / G$ is a fiber bundle projection with fiber $G / K$ and structure group $N(K) / K$.

Definition 1.2. A biaxial manifold $M$ is called $\pi$-biaxial if its bundle of principal orbits is trivial.

Let $G_{n}^{d}$ act on $R^{d n} \times R^{d n}$ via twice the standard representation. The orbit map $\pi_{d}: R^{d n} \times R^{d n} \rightarrow R^{d n} \times R^{d n} / G_{n}^{d}$ induces a functional structure on the orbit space, (i.e., a function $f$ on an open set $U \subset$ $R^{d n} \times R^{d n} / G_{n}^{d}$ to $R$ is smooth iff $f \circ \pi_{d}: \pi_{d}^{-1}(U) \rightarrow R$ is smooth). The following theorem is proved in Bredon [4].

THEOREM 1.1. The orbit space $R^{d n} \times R^{d n} / G_{n}^{d}$, with the functional structure induced from $\pi_{d}$, is diffeomorphic to the subset $K(d)$ of $R \times R^{d+1}$ consisting of those points $(y, a, b, c, d, e) \in R \times R^{d+1}$ such that $y \geqq\left(a^{2}+b^{2}+c^{2}+d^{2}, e^{2}\right)^{1 / 2}$ (the functional structure on $K(d)$ being the induced one from $\left.R \times R^{d+1}\right)$. The map that induces the diffeomorphism is

$$
\begin{gathered}
\bar{\pi}_{d}: R^{d n} \times R^{d n} \longrightarrow R \times R^{d+1} \\
(u, v) \longrightarrow\left(|u|^{2}+|v|^{2},|u|^{2}-|v|^{2}, 2\langle u, v\rangle\right) .
\end{gathered}
$$

Notice that $K(d)$ is a positive solid cone, diffeomorphic outside the origin to the half space $R^{+} \times R^{d+1}$.

2. Complex and quaternionic Stiefel manifolds. We shall now apply the results of the preceding section to the unitary and simplectic groups acting on the Stiefel manifold $V_{n+k+2,2}(C), V_{n+k+2,2}(Q)$ where $V_{n+k+2,2}(C)=U(n+k+2) / U(n+k), V_{n+k+2,2}(Q)=S p(n+k+2) /$ $S p(n+k)$. By $U(n) \subset U(n+k+2)(S p(n) \subset S p(n+k+2))$ we will denote the standard embedding of $U(n)(S p(n))$ into $U(n+k+2)$ $(S p(n+k+2))$, and by

$$
U(n): \frac{U(n+k+2)}{U(n+k)}\left(S p(n): \frac{S p(n+k+2)}{S p(n+k)}\right)
$$

the restriction to $U(n) \subset U(n+k+2)(S p(n) \subset S p(n+k+2))$ of the action given by left multiplication.

THEOREM 2.1. The Stiefel manifold $V_{n+k+2,2}(C)\left(V_{n+k+2,2}(Q)\right)$ is a $\pi$-biaxial $U(n)(S p(n))$ manifold. 
Proof. Let us observe that the $U(n)$ manifold $U(n+k+2) /$ $U(n+k)$ is an invariant submanifold of $C^{n} \times C^{n} \times C^{k+2} \times C^{k+2}$ with the representation $2 \sigma_{n}+\theta_{4 k+8}$, the submanifold being

$$
\begin{gathered}
V_{n+k+2,2}(C)=\left\{\left(v_{1}, v_{2}, w_{1}, w_{2}\right) \in C^{n} \times C^{n} \times C^{k+2} \times C^{k+2} /\right. \\
\left|v_{1}\right|^{2}+\left|w_{1}\right|^{2}=\left|v_{2}\right|^{2}+\left|w_{2}\right|^{2}=1
\end{gathered}
$$

and

$$
\left.\left\langle v_{1}, v_{2}\right\rangle_{c}+\left\langle w_{1}, w_{2}\right\rangle_{c}=0\right\} \text {. }
$$

The isotropy subgroup of $U(n)$ at $\left(v_{1}, v_{2}, w_{1}, w_{2}\right)$ is $U(n-i)$ if $\operatorname{dim}$ $\left\{v_{1}, v_{2}\right\}=i, \quad i=0,1,2$. Since $V_{n+k+2,2}(C)$ contains elements that satisfy any of the above conditions, the first condition of Definition 1.1 is satisfied. Using the implicit function theorem it is not hard to see that the tangent space to $V_{n+k+2,2}(C)$ at a fixed point, say $\left(0,0, w_{1}, w_{2}\right)$ is $U(n)$ equivalent (as $U(n)$ modules) to $\boldsymbol{C}^{n} \times \boldsymbol{C}^{n} \times$ $\boldsymbol{R}^{4 k+4}$, the $U(n)$ action on $\boldsymbol{C}^{n} \times \boldsymbol{C}^{n} \times \boldsymbol{R}^{4 k+4}$ given by $2 \sigma_{n}+\theta_{4 k+4}$. Similar arguments apply to the slice representations. Since $V_{n+k+2,2}(C)$ contains a principal orbit of $\boldsymbol{C}^{n} \times \boldsymbol{C}^{n} \times \boldsymbol{C}^{k+2} \times \boldsymbol{C}^{k+2}$, its bundle of principal orbits is a subbundle of that of $\boldsymbol{C}^{n} \times \boldsymbol{C}^{n} \times \boldsymbol{C}^{k+2} \times \boldsymbol{C}^{k+2}$ which is trivial (Theorem 1.1). Therefore $U(n+k+2) / U(n+k)$ is a $\pi$-biaxial $U(n)$ manifold. The proof in the simplectic case is completely analogous.

All the proofs in this section will be carried out for the unitary case since the simplectic case can be dealt with in a similar way.

Suppose that $\theta: G \times M \rightarrow M$ is a smooth action of a Lie group $G$ on a smooth manifold $M$. The orbit map $\pi: M \rightarrow M / G$ induces a functional structure on $M / G$ which we call the induced functional structure. Thus a function $f$ on an open set $U \subset M / G$ to $R$ is called smooth iff $f \circ \pi: \pi^{-1}(U) \rightarrow \boldsymbol{R}$ is smooth. From Theorem 1.1 it follows that the orbit space of a biaxial manifold with the induced functional structure is a smooth manifold except at the fixed point set.

The next theorem characterizes the orbit space of the $U(n)$ $(S p(n))$ action on $V_{n+k+2,2}(C)\left(V_{n+k+2,2}(Q)\right)$.

Let $X(\boldsymbol{C})(X(Q))$ denote the subspace of $\boldsymbol{C}^{k+2} \times \boldsymbol{C}^{k+2}\left(Q^{k+2} \times Q^{k+2}\right)$ consisting of all points $\left(w_{1}, w_{2}\right)$ such that, $\left|w_{1}\right|^{2} \leqq 1,\left|w_{2}\right|^{2} \leqq 1$ and $\left(1-\left|w_{1}\right|^{2}\right)\left(1-\left|w_{2}\right|^{2}\right)-\left|\left\langle w_{1}, w_{2}\right\rangle\right|^{2} \geqq 0$.

TheOREM 2.2. The orbit space of the $U(n)(S p(n))$ action on $V_{n+k+2,2}(C)\left(V_{n+k+2,2}(Q)\right)$ is diffeomorphic to $X(C)(X(Q))$. (The functional structure on $X(C)(X(Q))$ being the restriction of the $C^{\infty}$ one on $\boldsymbol{R}^{2 k+4} \times \boldsymbol{R}^{2 k+4}\left(\boldsymbol{R}^{4 k+8} \times \boldsymbol{R}^{4 k+8}\right)$.)

Proof. Clearly, the map $\phi: V_{n+k+2,2}(C) \rightarrow X(C), \phi\left(v_{1}, v_{2}, w_{1}, w_{2}\right)=$ 
$\left(w_{1}, w_{2}\right)$ induces a one to one morphism of functional structures, $\tilde{\phi}$ : $V_{n+k+2,2}(C) / U(n) \rightarrow X(C)$. To prove that $\tilde{\phi}$ is onto let $\left(w_{1}, w_{2}\right)$ be an element of $X(C)$, then by Theorem 1.1 there exist $\left(v_{1}, v_{2}\right) \in C^{n} \times C^{n}$ such that
(i ) $\left|v_{1}\right|^{2}+\left|v_{2}\right|^{2}=2-\left(\left|w_{1}\right|^{2}+\left|w_{2}\right|^{2}\right)$
(ii) $\left|v_{1}\right|^{2}+\left|v_{2}\right|^{2}=\left|w_{2}\right|^{2}-\left|w_{1}\right|^{2}$
(iii) $2\left\langle v_{1}, v_{2}\right\rangle=-2\left\langle w_{1}, w_{2}\right\rangle$.

But the above conditions imply that the element $\left(v_{1}, v_{2}, w_{1}, w_{2}\right)$ belongs to $V_{n+k+2,2}(C)$ and hence $\tilde{\phi}$ is surjective. Since in $C^{k+2} \times$ $C^{k+2}$ the $C^{\infty}$ functional structure is the same as the one induced from the projection $p_{2}: C^{n} \times C^{n} \times C^{k+2} \times C^{k+2} \rightarrow C^{k+2} \times C^{k+2}$ the theorem follows.

Note that the fixed point set of the $U(n)$ action on $V_{n+k+2,2}(C)$ is diffeomorphic to $U(k+2) / U(k)$ and the image under $\phi$ is the set

$$
\Sigma(\boldsymbol{C})=\left\{\left(w_{1}, w_{2}\right) \in C^{k+2} \times C^{k+2} /\left\langle w_{1}, w_{2}\right\rangle=0,\left|w_{1}\right|=\left|w_{2}\right|=1\right\} .
$$

Proposition 2.1. The subspace $X(C)(X(Q))$ is contractible.

Proof. It is not hard to check that the function $f: D^{4 k+8} \rightarrow$ $C^{k+2} \times C^{k+2}$ given by

$$
\begin{array}{rlrl}
f(x, y)=\left(\sqrt{\frac{-1+\sqrt{1+4 a}}{2 a}} x, \sqrt{\frac{-1+\sqrt{1+4 a}}{2 a} y}\right) a & \neq 0 \\
(x, y) & a & =0
\end{array}
$$

where $a$ denotes the real number $|\langle x, y\rangle|^{2}-|x|^{2}|y|^{2} /\left(|x|^{2}+|y|^{2}\right)^{2}$ is a homeomorphism onto $X(\boldsymbol{C})$.

3. The pullback construction. The main result of the theory of $\pi$-biaxial actions (Bredon [4]) gives a one to one correspondence between $G_{n}^{d} \pi$-biaxial manifolds with orbit space diffeomorphic to a fixed manifold $X$ and framed cobordism classes of framed submanifolds of $\partial X$ cobounding the fixed point set. As a consequence we have that to $U(n): U(n+k+2) / U(n+k)$ corresponds $\left[V^{4 k+5}, \mathscr{F}\right]$ where $\left[V^{4 k+5}, \mathscr{F}\right]$ denotes the framed cobordism class of a framed submanifold of $\partial(X(\boldsymbol{C}))$ cobounding $\Sigma(\boldsymbol{C})$.

Our objective now is to characterize $V^{4 k+5}$ and $\mathscr{F}$.

Let $\tau: C^{k+2} \times C^{k+2} \rightarrow R \times R \times C$

$$
\tau\left(w_{1}, w_{2}\right)=\left(2-\left(\left|w_{1}\right|^{2}+\left|w_{2}\right|^{2}\right),\left|w_{2}\right|^{2}-\left|w_{1}\right|^{2},-2\left\langle w_{1}, w_{2}\right\rangle\right) .
$$

It is easy to see that $\tau$ is transverse regular to zero therefore there exists a neighborhood about $\left(w_{1}, w_{2}\right) \in \Sigma(C)$ diffeomorphic to 
$U \times(\boldsymbol{R} \times \boldsymbol{R} \times \boldsymbol{C}), U$ open in $\Sigma(\boldsymbol{C})$, where $\tau$ is just the projection on the second factor. Now $X(C)=\tau^{-1}(K(2))$ where $K(2)$ is the image of $\bar{\pi}_{2}: \boldsymbol{C}^{n} \times \boldsymbol{C}^{n} \rightarrow \boldsymbol{R} \times \boldsymbol{R} \times \boldsymbol{C}(\S 1)$, so in $X(\boldsymbol{C})$, about $\left(w_{1}, w_{2}\right) \in \Sigma(\boldsymbol{C})$ we have a neighborhood diffeomorphic to $U \times K(2)$ where $\tau$ is just the projection.

Proposition 3.1. The map $\tau: \partial X \rightarrow \partial(K(2))$ is transverse regular to $R^{+}(1,1,0)$.

Proof. Transverse regularity is clear at zero so we may confine our attention to points $\left(w_{1}, w_{2}\right)$ such that $\left|w_{1}\right|^{2}<1,\left|w_{2}\right|^{2}=1$ and $\left\langle w_{1}, w_{2}\right\rangle=0$. For these points we observe that

$$
\begin{aligned}
& \gamma_{1}(t)=\left(w_{1}, w_{2}\right)\left(\begin{array}{r}
\cos t \sin t \\
-\sin t \cos t
\end{array}\right) \\
& \gamma_{2}(t)=\left(w_{1}, w_{2}\right)\left(\begin{array}{r}
\cos t i \sin t \\
-i \sin t \cos t
\end{array}\right)
\end{aligned}
$$

are curves in $\partial X$ such that the tangent vectors to $\tau \circ \gamma_{i} i=1,2$ at $t=0$ span the normal space at $\tau\left(w_{1}, w_{2}\right)$.

We will denote by $V^{4 k+5}$, the inverse image under $\tau$ of $R^{+}(1,1,0)$. Note that

$$
\begin{aligned}
V^{4 k+5}= & \left\{\left(w_{1}, w_{2}\right) \in C^{k+2} \times C^{k+2} /\left|w_{1}\right|^{2} \leqq 1,\right. \\
& \left.\left\langle w_{1}, w_{2}\right\rangle=0\right\}, \partial V^{4 k+5}=\Sigma(\boldsymbol{C}) .
\end{aligned}
$$

It follows from Proposition 3.1 that $V^{4 k+5}$ is a submanifold of $\partial X$ with trivial normal bundle.

Let $\mathscr{F}_{0}$ denote the standard framing of $R^{+}(1,1,0)$ in $\partial(K(2))$, $\mathscr{F}_{0}=\left\{X_{1}, X_{2}\right\}, X_{i}: R^{+}(1,1,0) \rightarrow \boldsymbol{R} \times \boldsymbol{R} \times \boldsymbol{C}, i=1,2, X_{1}(a, a, 0)=(0,0,1)$, $X_{2}(a, a, 0)=(0,0, i)$ and let $\mathscr{F}$ be the pullback by $\tau$ of $\mathscr{F}_{0}$, i.e., $(d \tau)(\mathscr{F})=\mathscr{F}_{0}$. The pullback $\mathscr{F}$ consists of smooth functions

$$
\begin{aligned}
& Y_{i}: V^{4 k+5} \longrightarrow C^{k+2} \times C^{k+2} \quad i=1,2 \\
& Y_{1}\left(w_{1}, w_{2}\right)=\frac{(-1)}{2\left(1+\left|w_{1}\right|^{2}\right)}\left(w_{2}, w_{1}\right) \\
& Y_{2}\left(w_{1}, w_{2}\right)=\frac{(-1)}{2\left(1+\left|w_{1}\right|^{2}\right)}\left(i w_{2},-i w_{1}\right) .
\end{aligned}
$$

It is not hard to see that $Y_{i}\left(w_{1}, w_{2}\right)$ is perpendicular to the tangent space of $V^{4 k+5}$ at the point $\left(w_{1}, w_{2}\right)$. Thus $\mathscr{F}$ defines a framing of $V^{4 k+5}$ (after dividing $Y_{i}$ by $\left\|Y_{i}\right\|$ ). Now it remains only to show 
that $\left(V^{4 k+5}, \mathscr{F}\right)$ is the framed submanifold of $\partial X$ cobounding $\Sigma(C)$ that corresponds to $U(n): U(n+k+2) / U(n+k)$.

By an analogous argument to the one in $\S 9$ of Bredon [2] it suffices to prove that the following diagram

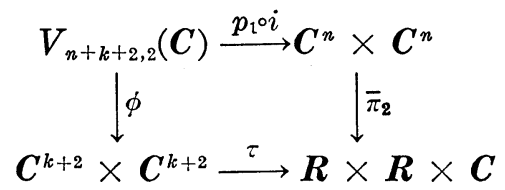

is a pullback diagram in the differential category. Here $\phi$ stands for projection on the last two coordinates and $\bar{\pi}_{2}$ is the map defined in $\S 1$. Clearly $V_{n+k+2,2}(C)$ is a pullback in the topological sense. Since the derivative of $\bar{\pi}_{2}$ is surjective except at the origin and $\tau$ is transverse regular to zero, the map $\bar{\pi}_{2} \times \tau$ is transverse regular to the diagonal. Hence the above diagram is a pullback diagram in the differential category.

Let $M$ be an $O(2 n)$ biaxial manifold. The restriction of $\sigma_{2 n}$ : $O(2 n) \rightarrow G l\left(\boldsymbol{R}^{2 n} \times \boldsymbol{R}^{2 n}\right)$ to $U(n) \subset O(2 n)$ is the standard representation $\sigma_{n}$ of $U(n)$. It follows that the restriction to $U(n)$ of an $O(2 n)$ biaxial manifold is a $U(n)$ biaxial manifold.

The most important result about restrictions of $\pi$-biaxial actions is proved in Bredon [4]. The theorem relates characteristic membranes (i.e., framed submanifolds of the boundary of the orbit space cobounding the fixed point set) of the $O(2 n)$ action and its restriction to $U(n)$. The statement of the theorem is the following:

THEOREM 3.1. If $M$ is an $O(2 n) \pi$-biaxial manifold such that $\left(M^{*}, M^{G}\right)$ is diffeomorphic to $(W, \Sigma)$, and the characteristic membrane is $V \subset \partial W$, then the restriction to $U(n)$ gives a $U(n) \pi$ biaxial manifold with orbit space diffeomorphic to $(W \times I, \Sigma)$ (corners straightened) and characteristic membrane $V \subset \partial W \subset \partial(W \times I)$ with the framing extended by the positive normal field of $\partial W$ in $\partial(W \times I)$.

Now we apply the theorem to our particular case.

We proved $(\S 2)$ that $((U(n+k+2)) / U(n+k) / U(n),(U(n+k+2) /$ $\left.U(n+k))^{U(n)}\right)$ was diffeomorphic to $(X(\boldsymbol{C}), \Sigma(\boldsymbol{C}))$.

Assume there exists a $\pi$-biaxial $O(2 n)$ action on $U(n+k+2) i$ $U(n+k)$. Let us denote by $Y$ the orbit space $(U(n+k+2)\}$ $U(n+k)) / O(2 n)$, with $Z$ the fixed point set $(U(n+k+2) / U(n+k))^{o(2 n)}$ and with $[\tilde{V}, \tilde{\mathscr{F}}]$ the characteristic membrane of the extension.

Because of Theorem 3.1 we have that, after smoothing the corners, $(Y \times I, Z)$ is the orbit space, and fixed point set of the 
restriction to $U(n)$ of the $O(2 n)$ action. But, if the $O(2 n)$ action is an extension of the original of $U(n): U(n+k+2) / U(n+k)$ then $(Y \times I, Z)$ is diffeomorphic to $(X(C), \Sigma(C))$. Moreover, the characteristic membrane for $U(n): U(n+k+2) / U(n+k)$ is frame cobordant to a framed submanifold of $\partial(Y)$.

In the next section we will assume that $Y$ is $D^{4 k+7}$, and we will contradict the existence of extension by proving the impossibility for $V^{4 k+5}$ of being frame cobordant to a submanifold of $S^{4 k+6}$.

4. Main theorem (complex case). This section contains the statement and proof of the main result for $U(n)$ acting on $V_{n+k+2,2}(C)$. We will study separately the cases $k=0$ and $k=2$ because in those cases $V^{4 k+5}$ is a trivial disk bundle. Then $k$ even $k \geqq 4$ and finally li odd.

Case $1 . \quad k=0$.

Proposition 4.1. The manifold $V^{5}$ is diffeomorphic to $D^{2} \times S^{3}$.

Proof. Let is denote the map

$$
\begin{aligned}
& r: D^{2} \times S^{3} \longrightarrow C^{2} \times C^{2} \\
& \gamma(z, q)=(z j q, q) .
\end{aligned}
$$

It is not hard to see that if gives the desired diffeomorphism.

In $\S 3$ we defined $\mathscr{F}(\partial X, \Sigma)$ as the set of framed cobordism classes of framed submanifolds of $\partial X$ cobounding $\Sigma(\boldsymbol{C})$. Now we are going to define a function

$$
\gamma_{\hat{o}, X}: \mathscr{F}(\partial X, \Sigma(\boldsymbol{C})) \longrightarrow\left[\partial X, S^{4}\right] .
$$

Let $\left[W^{5},\left(G_{1}, G_{2}\right)\right]$ be a framed submanifold of $\partial X$ cobounding $\Sigma(C)$. To this $\left[W^{5},\left(G_{1}, G_{2}\right)\right]$ we are going to associate a framed $S^{3}$ in $\partial X$ therefore an element of $\left[\partial X, S^{4}\right]$ (by the Thom-Pontryagin construction). We consider the embedding

$$
S^{3} \stackrel{\alpha}{\longrightarrow} S^{1} \times S^{3} \stackrel{\dot{\varphi}}{\longrightarrow} \Sigma(C) \longrightarrow \partial X
$$

where $\alpha(q)=(1, q)$ and $\gamma^{\prime}$ is the map of Proposition 4.1. The framing of $\psi^{\circ} \alpha: S^{3} \rightarrow \partial X$ being $G_{1}, G_{2},(d \psi)(1, q)(i, 0)$ and $N$. Here $N$ denotes the pointing out normal vector to $\Sigma(\boldsymbol{C})$ in $W^{5}$.

Let us consider, now, the following framed $S^{3}$ in $\partial X$. The embedding $\gamma$ defined by $\gamma(q)=(0, q)$ and the framing by $Z_{1}(0, q)=(q, 0)$, $Z_{2}(0, q)=(i q, 0), Z_{3}(0, q)=(j q, 0), Z_{4}(0, q)=(k q, 0)$. This framed $S^{3}$ 
in $\partial X$ defines an element $[\alpha]$ of $\left[\partial X, S^{4}\right]$ and it is not hard to see that $[\alpha]=\gamma_{\partial X}\left[\left(V^{5},\left(Y_{1}, Y_{2}\right)\right)\right]$. After these considerations we are ready to prove the main result for $k=0$.

THEOREM 4.1. The action of $U(n)$ on $U(n+2) / U(n)$ can not be extended to a biaxial $O(2 n)$ action with orbit space diffeomorphic to $D^{8}$.

Proof. Assume there exists a $\pi$-biaxial action

$$
O(2 n) \times \frac{U(n+2)}{U(n)} \longrightarrow \frac{U(n+2)}{U(n)}
$$

which extends the $U(n)$ action on $U(n+2) / U(n)$ and with the property $(U(n+2) / U(n)) / O(2 n) \approx D^{8}$. Then by the discussion in $\S 3$, $\left[V^{5},\left(Y_{1}, Y_{2}\right)\right]$ is frame cobordant to a submanifold of $S^{6} \subset \partial X \approx S^{7}$. Then the element $\gamma_{\partial X}\left[\left(V^{5},\left(Y_{1}, Y_{2}\right)\right)\right]$ is in the image of the suspension homomorphism $s: \pi_{6}\left(S^{3}\right) \longrightarrow \pi_{7}\left(S^{4}\right)$. We want to prove that this is impossible. Let us consider the following diagram

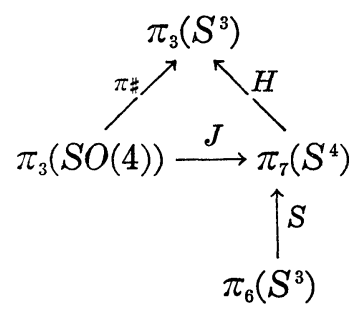

where $J$ is the Hopf-Whitehead homomorphism and $H$ is the Hopf invariant.

Since $\gamma_{\partial X}\left[V^{5},\left(Y_{1} Y_{2}\right)\right]=J([R])$ where $R(q)$ is right multiplication by $q, q \in S^{3}$, we get a contradiction because $H \circ S=0$ but $\pi_{\ddagger}([R])$ is not.

Case 2. $k=2$.

Let $i$ and $\alpha$ denote the following maps

$$
\begin{array}{ll}
i: & \boldsymbol{R}^{6} \rightarrow \boldsymbol{R}^{8}=\boldsymbol{R}^{2} \times \boldsymbol{R}^{6} \quad i(x)=(0, x) \\
\alpha: & \boldsymbol{C}^{4} \rightarrow \boldsymbol{C}^{4} \quad \alpha\left(z_{1}, z_{2}, z_{3}, z_{4}\right)=\left(z_{1}, z_{2}, z_{3}, \bar{z}_{4}\right),
\end{array}
$$

Using the Cayley multiplication we define

$$
\begin{aligned}
& \phi: D^{6} \times S^{7} \longrightarrow C^{4} \times C^{4} \\
& \phi(x, y)=(\alpha((i x) y), \alpha(y)) .
\end{aligned}
$$

The map $\phi$ is a diffeomorphism onto $V^{13}$.

As in the case $k=0$ we define

$$
\gamma_{\partial X}: \mathscr{F}(\partial X, \Sigma) \longrightarrow\left[\partial X, S^{8}\right]
$$


and with analogous proof we have that $\gamma_{\partial X}\left(\left[V^{13},\left(Y_{1}, Y_{2}\right)\right]\right)=J([R])$ $\langle\boldsymbol{R}(x)$ right (Cayley) multiplication by $x)$ can not be in the image of the suspension homomorphism. Therefore we have

THEOREM 4.2. The $U(n)$ action on $U(n+4) / U(n+2)$ can not be extended to a biaxial $O(2 n)$ action with orbit space diffeomorphic to $D^{16}$.

Case 3. $k$ even, $k \geqq 4$.

Let $p: V^{4 k+5} \rightarrow S^{2 k+3}$ be the projection on the second factor. Note that when $k$ is even we have a section

$$
\begin{gathered}
s: S^{2 k+3} \longrightarrow \Sigma^{4 k+4} \\
s\left(\left(z_{1}, z_{2}, \cdots, z_{k+2}\right)\right)=\left(\left(\bar{z}_{2},-z_{1}, \bar{z}_{4},-\bar{z}_{3}, \cdots\right)\left(z_{1}, z_{2}, \cdots, z_{k+2}\right)\right) .
\end{gathered}
$$

It is not hard to see that the map $p: V^{4 k+5} \rightarrow S^{2 k+3}$ is a disk bundle projection characterized by a generator of $\pi_{2 k+2}(S O(2 k+2)) \approx Z_{4}$.

THEOREM 4.3. The action of $U(n)$ on $U(n+k+2) / U(n+k), k$ even $k \geqq 4$ can not be extended to a biaxial $O(2 n)$ action with orbit space diffeomorphic to $D^{4 k+8}$.

Proof. Assume there exists such an extension. Then $\left(V^{4 k+5}\right.$, $\left.\left(Y_{1}, Y_{2}\right)\right)$ is frame cobordant to $\left(\widetilde{V}^{4 k+5},\left(\widetilde{Y}_{1}, \widetilde{Y}_{2}\right)\right)$, where $\widetilde{V}^{4 k+5}$ is a framed submanifold of $S^{4 k+6} \subset \partial X \approx S^{4 k+7}$.

Therefore the normal bundle of

$$
S^{2 k+3} \stackrel{s}{\longrightarrow} \Sigma^{4 k+4} \longrightarrow \widetilde{V}^{4 k+5} \longrightarrow S^{4 k+k}
$$

is represented by a generator of $\pi_{2 k+2}(S O(2 k+3))$ which is $Z_{2}$ for $k$ even, $k \geqq 4$ (Kervaire [11]). This contradicts the known fact (see Haefliger [6]) that any $S^{2 k+3}$ embedded in $S^{4 k+6}$ is unknotted.

We observe that from the above proof and Theorem 11.2 (Bredon [4]) it follows that there does not exist, for $n+k$ even, $n+k \geqq 4$ a biaxial $O(2 n)$ action on $V_{n+k+2,2}(\boldsymbol{C})$ with orbit space diffeomorphic to a disk.

Case $4 . \quad k$ odd.

This case follows easily from the even one.

CoROLlaRY 4.1. The action of $U(n)$ on $U(n+k+2) / U(n+k)$, $n \geqq 3, k$ odd can not be extended to a biaxial $O(2 n)$ action with orbit space diffeomorphic to a disk. 
Proof. If $U(n): U(n+k+2) / U(n+k)$ were the restriction of an $O(2 n)$ biaxial action then $U(n-1): U(n-1+(k+1)+2) / U(n-$ $1+(k+1)$ ) would be the restriction of an $O(2 n-2)$ biaxial action, but this is a contradiction because $k+1$ is even.

REMARK 1. In the preceding theorems we assumed that the $O(2 n)$ biaxial extension had orbit space diffeomorphic to a disk. It can be proved that this is no restriction. In fact, if $U(n): U(n+$ $k+2) / U(n+k)$ is the restriction of a biaxial $O(2 n)$ action, $U(n-1)$ : $U(n-1+(k+1)+2) / U(n-1+(k+1))$ is the restriction of a biaxial $O(2 n-2)$ action, but since the orbit spaces of the $U(n)$ and $U(n-1)$ actions are disks we have that the orbit spaces of the $O(2 n)$ and $O(2 n-2)$ actions are contractible. Following the same kind of arguments as in Bredon [4], Chapter $V$ we can prove that the boundary of $U(n-1+(k+1)+2) / U(n-1+(k+1)) / O(2 n-2)$ is simply connected, hence it is a disk. Therefore we get a contradition.

REMARK 2. Since the classification theorem for $S O(n)$ biaxial actions is the same that the one for $O(n), n>3$, in the preceding theorems was proved that the $U(n)$ action on $V_{n+k+2,2}(C)$ can not be extended to a biaxial $S O(2 n)$ action. A natural question is the following: Can the $U(n)$ action on $V_{n+k+2,2}(C)$ be extended to $S O(2 n)$ ? The next proposition answer almost completely the question.

Proposition 4.3. Given $k, n$, such that $n^{2}-5 n>4 k+4$ the $U(n)$ action on $V_{n+k+2,2}(C)$ can not be extended to $S O(2 n)$ (therefore to $O(2 n))$.

Proof. Suppose there exists such an extension and let $x$ be a fixed point for the $U(n)$ action. Then the connected component of the isotropy subgroup of $x,\left(S O(2 n)_{x}\right)^{0}$, contains the maximal torus of $S O(2 n)$. Let $Z$ be the center of $\left(S O(2 n)_{x}\right)^{0}$, then

$$
\left(S O(2 n)_{x}\right)^{0}=\left(N_{S O(2 n)}(Z)\right)^{0} \text { see Borel [2] }
$$

where $N_{S O(2 n)}(Z)$ denotes the normalizer of $Z$ in $S O(2 n)$.

The center $Z$ must be $Z_{2}$ (therefore $x$ fixed) otherwise $\left(S O(2 n)_{x}\right)^{0}$ would be $U(n)$ which is impossible by dimensional reasons.

Now, the tangential representation at $x$ of $S O(2 n)$ must be $2 \sigma_{2 n}+\theta_{4 k+4}$ because restricted to $U(n)$ is $2 \sigma_{n}+\theta_{4 k+4}$ therefore the principal orbit type is $S O(2 n) / S O(2 n-2)$. After these considerations the biaxiality of the $S O(2 n)$ action follows from a theorem of Hsiang (see Hsiang [8]), therefore a contradiction. 
5. Biaxial $S p(n)$ manifolds. Let us consider the left translation action of $S p(n) \subset S p(n+k+2)$ on the quaternionic Stiefel manifold $S p(n+k+2) / S p(n+k)$. It is not hard to prove, using the same kind of arguments as the ones we used to prove the results of $\S 3$, the following theorem.

THEOREM 5.1. Let

$$
\begin{aligned}
& V^{8 k+11}=\left\{\left(w_{1}, w_{2}\right) \in Q^{k+2} \times Q^{k+2} /\left|w_{1}\right|^{2} \leqq 1,\left|w_{2}\right|^{2}=1,\left\langle w_{1}, w_{2}\right\rangle=0\right\} \\
& \mathscr{F}=\left\{Y_{i}\right\}_{i=1}^{4} \quad Y_{i}: V^{4 k+11} \longrightarrow \boldsymbol{R}^{8 k+8} \times \boldsymbol{R}^{8 k+8} \\
& Y_{i}\left(w_{1}, w_{2}\right)=\left(q_{i} w_{2}, \bar{q}_{i} w_{1}\right) \quad q_{i}= \begin{cases}1 & i=1 \\
i & i=2 \\
j & i=3 \\
k & i=4\end{cases}
\end{aligned}
$$

Then, the cobordism class of the pair $\left(V^{8 k+11}, \mathscr{F}\right)$ is the element that corresponds (via Theorem 13.3, Bredon [4]) to the action of $S p(n)$ on $S p(n+k+2) / S p(n+k)$.

Assume that the action of $S p(n)$ on $S p(n+k+2) / S p(n+k)$ can be extended to a $\pi$-biaxial action of $U(2 n)$ such that the orbit space $S p(n+k+2) / S p(n+k) / U(2 n)$ is homeomorphic to $D^{8 k+16}$. Since we are dealing with biaxial actions, the fixed point sets of the $S p(n)$ action and its extension to $U(2 n)$ are the same. Let $\left[\left(E_{0}^{8 k+11}, \mathscr{G}_{0}\right)\right]$ denote the cobordism class of the framed submanifold of $S^{8 k+13}$ cobounding the fixed point set that corresponds to the $U(2 n)$ action. Then, by the analog of Theorem 3.1, the restriction to $S p(n)$ gives a $S p(n) \pi$-biaxial manifold with orbit space diffeomorphic to $D^{8 k+16}$ and characteristic membrane $\left(E_{0}^{8 k+11}, \tilde{\mathscr{G}}_{0}\right)$ where $\tilde{\mathscr{G}}_{0}$ is $\mathscr{G}_{0}$ plus the restriction to $E_{0}^{8 k+11}$ of the standard framing of $S^{8 k+13} \subset S^{8 k+15}$. Since we are assuming that the restriction to $S p(n)$ is equivalent to the original action, we have that $\left[\left(V^{8 k+11}, \mathscr{F}\right)\right]=$ $\left[\left(E_{0}^{8 k+11}, \tilde{\mathscr{G}}\right)\right]$ (the brackets denote framed cobordism classes). Our main objective is to show that the last equality gives us a contradiction. We cannot use here the same kind of argument we used in $\S 4$ for the $U(n)$ case, because, even when $V^{8 k+11}$ is still a disk bundle over a sphere we do not have (at least for an infinite family of $k$ 's) nontrivial cross sections (see James [9], [10]).

Let $\left(E_{0}^{8 k+11}, \tilde{\mathscr{G}}_{0}\right)$ be a connected framed submanifold of $S^{8 k+14}$ with boundary $\partial E_{0}$ diffeomorphic to $S p(k+2) / S p(k)$.

Proposition 5.1. The pair $\left(E_{0}^{8 k+11}, \tilde{\mathscr{G}}_{n}\right)$ is frame cobordant to a $4 k+5$ connected pair $\left(E^{8 k+11}, \mathscr{G}\right)$. 
Proof. The technique for simplifying $\left(E_{0}, \tilde{\mathscr{G}}_{0}\right)$ will be as to be expected, that of framed spherical modifications as presented in Haefliger [6] (Proposition 3.3).

Let $\alpha \in \pi_{r}\left(E_{0}\right)$, then the hypotheses of Haefliger [6] are:

(1) $\alpha$ is represented by an imbedded sphere $s$ in $\operatorname{int} E_{0}$.

(2) $s$ bounds an imbedded disk $d$ in $S^{8 k+14}$ such that $d \cap E_{0}=s$ and $f_{1} \mid s$ points radially into $d\left(\tilde{\mathscr{G}}_{0}=\left\{f_{i}\right\}_{i=1}^{3}\right)$.

(3) the frame $\left(f_{2}, f_{3}\right) \mid s$ extends to a partial normal frame on $d$.

The proof of Proposition 3.3, Haefliger [6], shows that if these hypotheses are satisfied $\left(E_{0}, \tilde{\mathscr{G}}_{0}\right)$ is frame cobordant to $\left(\chi\left(E_{0}, f\right)\right.$, $\left.\chi\left(\tilde{\mathscr{G}}_{0}\right)\right)$ for some imbedding $f: S^{r} \times D^{8 k+11-r} \rightarrow E_{0}$ representing $\alpha$. Assume $\left(E_{0}^{8+11}, \tilde{\mathscr{G}}_{0}\right)$ is $r-1$ connected, $0<r \leqq 4 k+5$. We will show that hypotheses (1), (2) and (3) can be satisfied for all $\alpha \in \pi_{r}\left(E_{0}\right)$; then will follow from Milnor [13] and Haefliger [6] that $\left(E_{0}, \tilde{\mathscr{G}}_{0}\right)$ is frame cobordant to a $4 k+4$ connected manifold. In Kervaire and Milnor [12], Kervaire and Milnor show how to kill the middle homotopy group when the boundary of the manifold is a homology sphere. The same proof works here because of the fact that the middle dimension homology of the boundary is zero, therefore the proposition will follow.

Hypothesis (1) is a consequence of classical results of Whitney together with the fact that $E_{0}^{8 k+11}$ is parallelizable.

Hypothesis (2) will follow if $S^{8 k+14}-E_{0}^{8 k+11}$ is $r$-connnected. By Lefschetz duality $H_{0}\left(S^{8 k+14}-E_{0}\right) \approx H^{8 k+14}\left(S^{8 k+14}, E_{0}\right) \approx Z$. The 1-connectedness of $S^{8 k+14}-E_{0}$ follows from the 1-connectedness of $S^{8 k+14}$ by a general position argument (Milnor [14]). It is not hard to check that $H_{i}\left(S^{8 k+14}-E_{0}\right)=0$ for $2 \leqq i \leqq r$; an application of the Hurewicz theorem then shows that $S^{8 k+14}-E_{0}$ is $r$-connected.

Hypothesis (3) is trivially satisfied because the obstruction to extending $\left(f_{2}, f_{3}\right) \mid s$ over $d$ is represented by an element

$$
\xi \in \pi_{r}\left(V_{8 k+13-r, 2}\right) \text {. }
$$

Since $2 r<8 k+11$ this group is zero (Browder [5]). Hence the proposition follows.

Let $(W, \mathscr{C})$ denote the framed cobordism between $\left(V^{8 k+11}, \mathscr{F}\right)$ and the $4 k+5$ connected submanifold of $S^{8 k+14}$ (Proposition 5.1) $\left(E^{8 k+11}, \mathscr{G}\right)$.

Applying the technique of framed spherical modifications to $(W, \mathscr{C})$ we may assume that the pair $(W, \mathscr{H})$ is $4 k+5$ connected. The obstruction to make it $4 k+6$ connected is its signature and it 
is easy to see that it is a multiple of eight.

THEOREM 5.2. There exists a framed h-cobordism between $\left(V^{8 k+11}, \mathscr{F}\right)$ and a framed submanifold of $S^{8 k+14}$.

Proof. Assume sign $(W)=8 \gamma$. It follows from the results of Browder [5] that there exists a $2(2 k+3)-1$ connected $\pi$-manifold $M^{8 k+12}$ with $\partial M$ a homotopy sphere such the $\operatorname{sign}(M)=8 \gamma$. According to Hirsch [1], $M$ embeds in $S^{8 k+13}$ and since $M$ is a $\pi$-manifold, $M$ embeds in $S^{8 k+13}$ with normal frame $\mathscr{F}_{M}$.

Let us consider $M$ embedded in $S^{8 k+15} \times I$ with the property $M \cap\left(S^{8 k+15} \times\{0\}\right)=\partial M$. The connected sum along the boundary (Milnor and Kervaire [12]) give us a new framed submanifold $(\tilde{W}, \tilde{\mathscr{H}}), \quad \tilde{\mathscr{H}}=\left\{\widetilde{h}_{i}\right\}_{i=1}^{4}$ of $S^{8 k+15} \times I$, which is $4 k+5$ connected, has signature zero and is a framed cobordism between $V^{8 k+11}$ and $E^{8 k+11} \sharp \partial M$. It follows from Milnor [13] that $\pi_{4 k+6}(\tilde{W})$ may be killed by a sequence of spherical modifications on $W$ associated with elements of $\pi_{4 k+6}(\widetilde{W})$ whose self intersection number is zero. That hypothesis (3) of Proposition 3.3, Haefliger [6], is satisfied for all such elements follows from the fact that the obstruction to extending $\widetilde{h}_{i} \mid s, i=2,3,4$ over $d$ is represented by $\xi \in \pi_{4 k+6}\left(V_{4 k+9,3}\right)$. But if

$$
\partial: \pi_{4 k+6}\left(V_{4 k+9,3}\right) \longrightarrow \pi_{4 k+5}(S O(4 k+6))
$$

is the boundary homomorphism of the fibration $S O(4 k+6) \rightarrow$ $S O(4 k+9) \rightarrow V_{4 k+9,3}$ then $\partial(\xi)$ corresponds to the normal bundle of $s$ according to Haefliger [6]; then by Lemma 20, Milnor [13], $\partial(\xi)=0$.

According to Kervaire [11]

$$
\pi_{4 k+6}(S O(4 k+9))= \begin{cases}0 & k \text { even } \\ Z & k \text { odd }\end{cases}
$$

and according to Paechter [16] $\pi_{4 k+6}\left(V_{4 k+9,3}\right) \approx Z$; then $\partial$ is a monomorphism and $\xi=0$. Therefore the spherical modifications can be carried out on $(\tilde{W}, \tilde{\mathscr{C}})$ and we get a framed $4 k+6$ connected cobordism (that we will denote again by $(\tilde{W}, \tilde{\mathscr{C}})$ ) between $\left(V^{8 k+11}\right.$, $\mathscr{F})$ and $\left(E^{8 k+11}, \mathscr{G}\right) \#\left(\partial M, \mathscr{F}_{M} \mid \partial M\right)=\left(\widetilde{E}^{8 k+11}, \tilde{\mathscr{E}}\right)$. It is not hard to show that the homomorphisms

$$
\begin{aligned}
& i_{*}: H_{4 k+7}\left(V^{8 k+11}\right) \longrightarrow H_{4 k+7}(\widetilde{W}) \\
& i_{*}: H_{4 k+7}\left(\widetilde{E}^{8 k+11}\right) \longrightarrow H_{4 k+7}(\widetilde{W})
\end{aligned}
$$

are isomorphisms; therefore $\tilde{W}$ is an $h$-cobordism and the theorem follows. 
We are ready now to prove the main theorem of this section.

THEOREM 5.3. The $S p(n)$ action on $S p(n+k+2) / S p(n+k)$ can not be extended to a $\pi$-biaxial $U(2 n)$ action with orbit space diffeomorphic to a disk.

Proof. We know that if such an extension exists, we have a framed $h$-cobordism between $\left(V^{8 k+11}, \mathscr{F}\right)$ and a framed submanifold of $S^{8 k+14}$. If we restrict $\mathscr{F}$ to $S^{4 k+7} \subset V^{8 k+11}$ we have an element $[\alpha] \in \pi_{4 k+7}\left(V_{4 k+8,4}\right)$ where

$$
\alpha(X)=((x, 0)(i x, 0)(j x, 0)(k x, 0)) .
$$

But then the element $[\alpha]$ is in the image of the homomorphism

$$
i_{\sharp}: \pi_{4 k+7}\left(V_{4 k+7,3}\right) \longrightarrow \pi_{4 k+7}\left(V_{4 k+8,4}\right)
$$

which is impossible, because $q_{\sharp}[\alpha]$ is a generator of $\pi_{4 k+7}\left(S^{4 k+7}\right)$. (Here $q=V_{4 k+8,4} \rightarrow S^{4 k+7}$ denotes the projection onto the last factor). Hence we proved impossibility of such an extension.

REMARK. In Theorem 5.3 we assumed that the orbit space of the $U(2 n)$ extension was a disk, but this is no restriction. The proof is similar to the ortogonal case $(\S 4)$.

\section{REFERENCES}

1. F. Adams, On the groups $J(x), I V$, Topolopy, vol. 5 (1966).

2. A. Borel, and J. De Siebenthal, Les sous groups fermés de rang maximum des groupes de Lie clos, Comment Math. Helve, (1949).

3. G. Bredon, Introduction to Compact Transformation Groups, Academic Press, New York, 1972.

4. - Biaxial Actions of the Classical Groups, Xerox Copies-Rutgers University. 5. W. Browder, Surgery on Simply Connected Manifolds, Springer Verlag, New York, 1972.

6. A. Haefliger, Knotted $4 k-1$ spheres in $6 k$ space, Annals of Math., vol. 75.

7. B. Harris, Some calculations of homotopy groups of symmetric spaces, Trans, Amer. Math Soc., (1963).

8. Wu. Y. Hsiang, Cohomology Theory of Topological Transformation Groups, Springer Verlag, New York, 1975.

9. I. James, Cross sections of Stiefel manifolds, Proc. London Math. Soc., (3), (1958).

10. - Spaces associated with Stiefel manilfolds, Proc. London Math. Soc., (1959).

11. M. Kervaire, Some nonstable homotopy groups of Lie groups, Illinois J. Math., 4 (1960).

12. M. Kervaire and J. Milnor, Groups of homotopy spheres I, Ann. of Math., 77 (1963).

13. J. Milnor, A procedure for killing the homotopy groups of differentiable manifolds, Symposia in Pure Mathematics, Amer. Math. Soc., 3 (1961).

14. Lectures on the h-cobordism Theorem, Princeton University Press, Princeton, 1965. 
15. J. Milnor, On simply connected 4-manifolds, Symposium Internacional de Topologia Algebraica, Mexico, (1958).

16. G. Paechter, The groups $\pi_{r}\left(V_{n, m}\right)$, Quart. J. Math. Oxford Ser (2), 7 (1956).

Received February 6, 1979. The results of this article are part of the $\mathrm{Ph}$. D. thesis of the author written under the direction of professor Glen Bredon at Rutgers University.

Universidade Federal de Pernambuco

50.000 RECIFE PE

BRAZIL 



\section{PACIFIC JOURNAL OF MATHEMATICS}

\section{EDITORS}

DONALD BABBITT (Managing Editor)

University of California

Los Angeles, California 90024

Hugo RossI

University of Utah

Salt Lake City, UT 84112

C. C. MOORE and ANDrew OGG

University of California

Berkeley, CA 94720
J. DUgundjI

Department of Mathematics University of Southern California Los Angeles, California 90007

R. FinN aNd J. Milgram Stanford University Stanford, California 94305

\section{ASSOCIATE EDITORS}

E. F. BECKENBACH

B. H. NeUMANN

F. WOLF

K. YosHIDA

\section{SUPPORTING INSTITUTIONS}

UNIVERSITY OF BRITISH COLUMBIA CALIFORNIA INSTITUTE OF TECHNOLOGY UNIVERSITY OF CALIFORNIA MONTANA STATE UNIVERSITY UNIVERSITY OF NEVADA, RENO NEW MEXICO STATE UNIVERSITY OREGON STATE UNIVERSITY UNIVERSITY OF OREGON
UNIVERSITY OF SOUTHERN CALIFORNIA STANFORD UNIVERSITY UNIVERSITY OF HAWAII UNIVERSITY OF TOKYO UNIVERSITY OF UTAH WASHINGTON STATE UNIVERSITY UNIVERSITY OF WASHINGTON 


\section{Pacific Journal of Mathematics}

\section{Vol. 84 , No. 1 \\ May, 1979}

Michael James Beeson, Goodman's theorem and beyond ...............

Robert S. Cahn and Michael E. Taylor, Asymptotic behavior of multiplicities

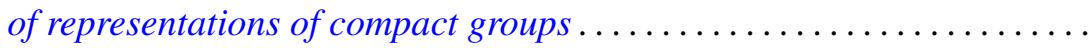

Douglas Michael Campbell and Vikramaditya Singh, Valence properties of

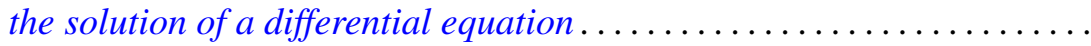

J.-F. Colombeau, Reinhold Meise and Bernard Perrot, A density result in spaces of Silva holomorphic mappings .....................

Marcel Erné, On the relativization of chain topologies .................

Le Baron O. Ferguson, Uniform and $L_{p}$ approximation for generalized

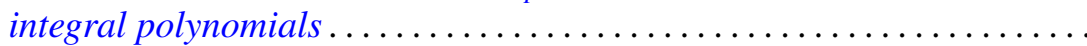

Kenneth R. Goodearl and David E. Handelman, Homogenization of regular rings of bounded index..................................

Friedrich Haslinger, A dual relationship between generalized

Abel-Gončarov bases and certain Pincherle bases .................

Miriam Hausman, Generalization of a theorem of Landau. .

Makoto Hayashi, 2-factorization in finite groups

Robert Marcus, Stochastic diffusion on an unbounded domain ........... 143

Isabel Dotti de Miatello, Extension of actions on Stiefel manifolds....

C. David (Carl) Minda, The hyperbolic metric and coverings of Riemann surfaces...

Somashekhar Amrith Naimpally and Mohan Lal Tikoo, On $T_{1}$-compactifications.

Chia-Ven Pao, Asymptotic stability and nonexistence of global solution for a semilinear parabolic equation ....

Shigeo Segawa, Harmonic majoration of quasibounded type ...

Sze-Kai Tsui and Steve Wright, The splitting of operator algebras ...

Bruce Williams, Hopf invariants, localization and embeddings of Poincaré complexes....

Leslie Wilson, Nonopenness of the set of Thom-Boardman maps ...

Alicia B. Winslow, There are $2^{\mathrm{c}}$ nonhomeomorphic continua in $\beta R^{n}-R^{n}$ 